NISTIR 8159

\title{
Usability Testing of a Contactless Fingerprint Device: Part 2
}

\author{
Brian Stanton \\ Mary Theofanos \\ Susanne Furman \\ Patrick J. Grother
}

This publication is available free of charge from:

https://doi.org/10.6028/NIST.IR.8159

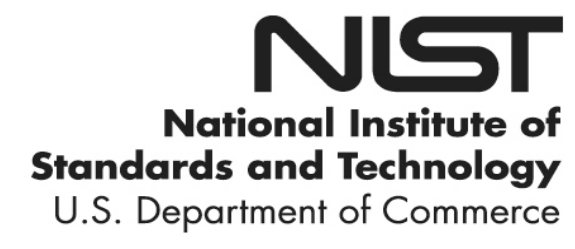


NISTIR 8159

\title{
Usability Testing of a Contactless Fingerprint Device: Part 2
}

\author{
Brian Stanton \\ Mary Theofanos \\ Susanne Furman \\ Patrick Grother \\ Information Access Division \\ Information Technology Laboratory
}

This publication is available free of charge from:

https://doi.org/10.6028/NIST.IR.8159

December 2016

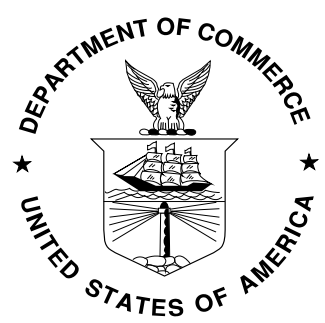

U.S. Department of Commerce Penny Pritzker, Secretary

National Institute of Standards and Technology Willie May, Under Secretary of Commerce for Standards and Technology and Director 


\section{Table of Contents}

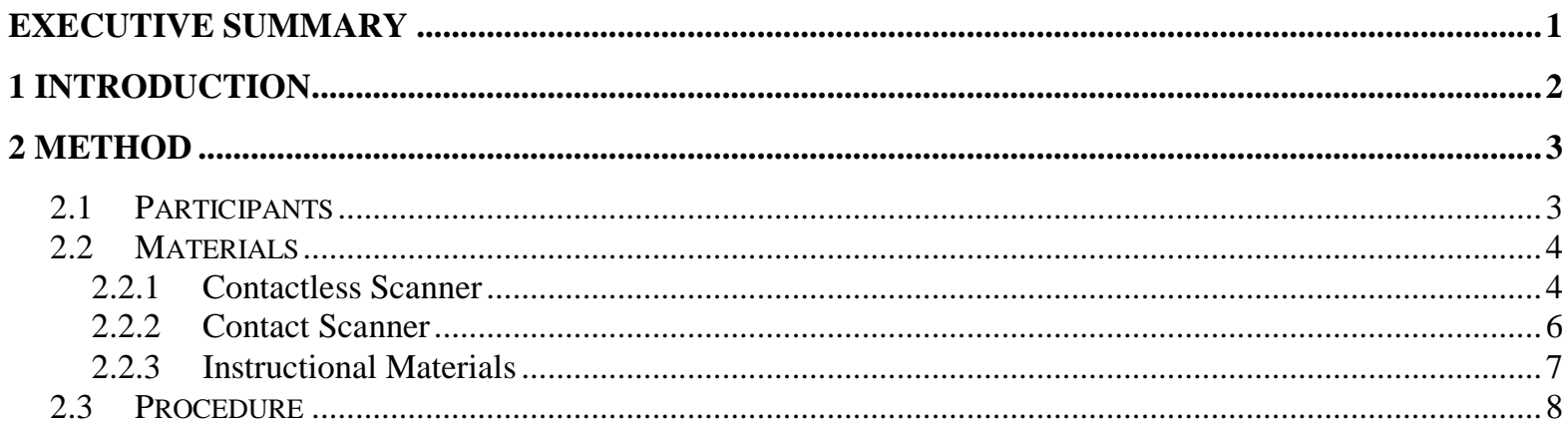

3 RESULTS

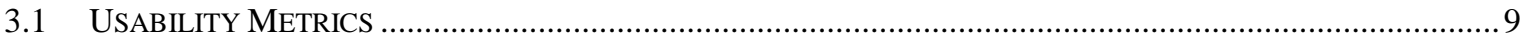

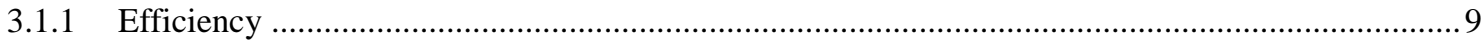

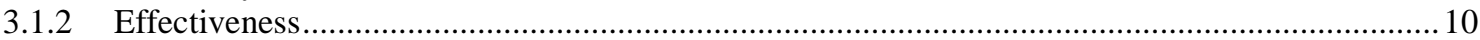

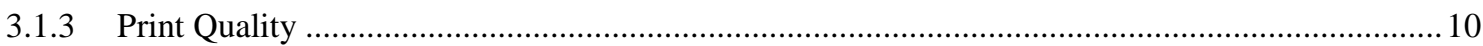

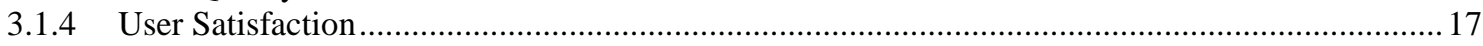

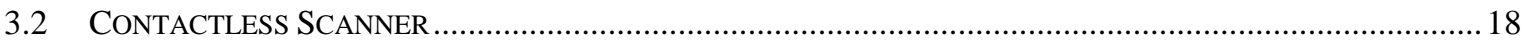

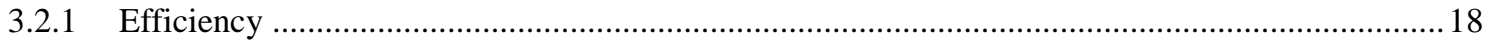

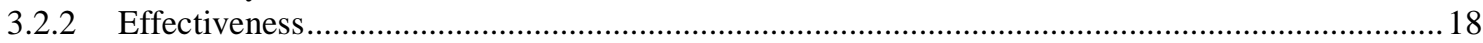

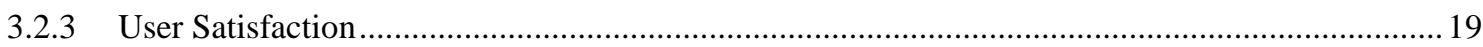

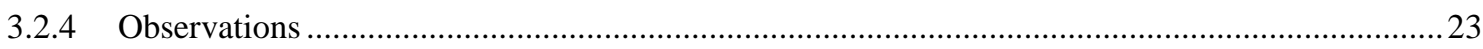

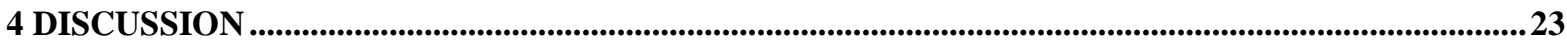

4.1 MENTAL ModELS OF FINGERPRINTING - TOUCHING THE GLASS ................................................23

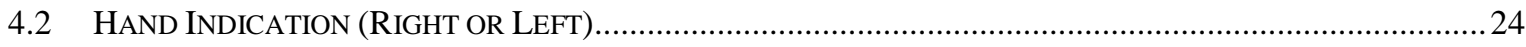

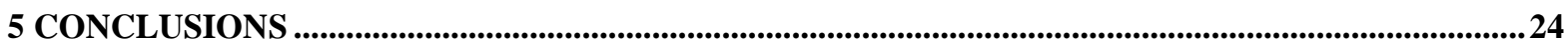

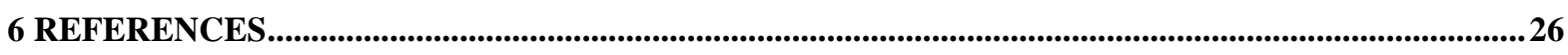

APPENDIX A: DEMOGRAPHICS QUESTIONNAIRE........................................................................27

APPENDIX B: POST-TASK QUESTIONNAIRE....................................................................................... 28 


\section{$\underline{\text { List of Figures }}$}

FIGURE 1: AGE RANGE OF CONTACTLESS SCANNER PARTICIPANTS .............................................................. 4

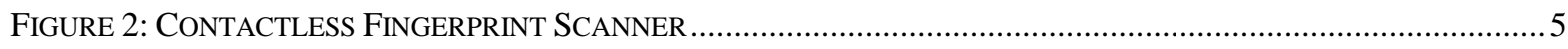

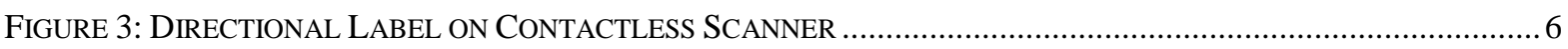

FIGURE 4: TRADITIONAL FINGERPRINT SCANNER WITH INDICATORS..............................................................

FigURE 5: TRADITIONAL “FingERPRINT SCANNER SETUP SHOWING MAT AND ADJUSTABLE TABLE..................... 9

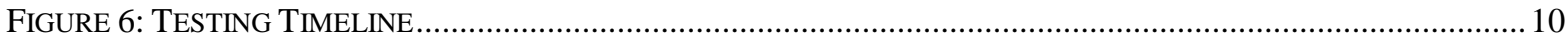

FIGURE 7 - DATA OUTPUTS AND PROCESSING FROM CONTACT OPTICAL SCANNER ............................................... 11

FIGURE 8 - DATA OUTPUTS FROM CONTACTLESS SCANNER AS CONFIGURED FOR NIST TRIAL..............................11 


\section{$\underline{\text { List of Tables }}$}

TABLE 1 - COUNTS OF SUBJECTS AND PRESENTATIONS USED IN THE ANALYSIS ................................................. 12

TABLE 2 - COUNTS OF N-FINGER CAPTURES USING THE CONTACTLESS SCANNER …........................................... 13

TABLE 3 - DURATIONS OF N-FINGER CAPTURES USING THE CONTACTLESS SCANNER .........................................13

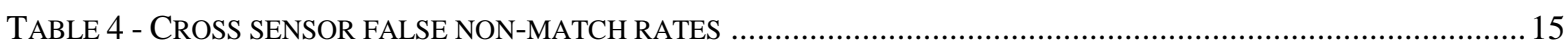

TABLE 5 - NUMBER OF PERSONS UNABLE TO AUTHENTICATE OVER REPEATED ATTEMPTS.................................... 16

TABLE 6: Average TASK TimeS IN SECONDS - ConTACTLESS SCANNER ….................................................... 18

TABLE 7: SUCCESSFUL PRINT COLLECTION PER TASK - CONTACTLESS SCANNER ............................................... 19

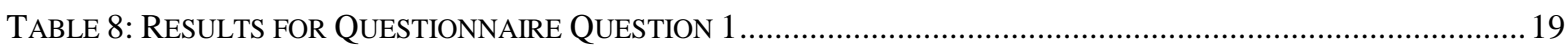

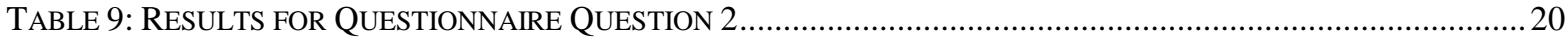

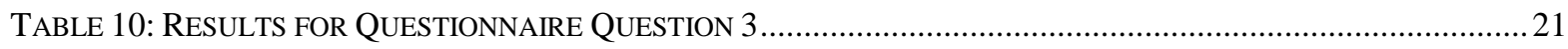

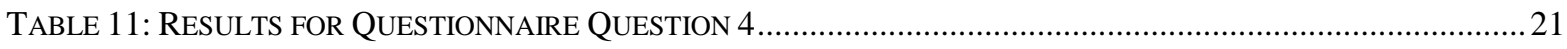

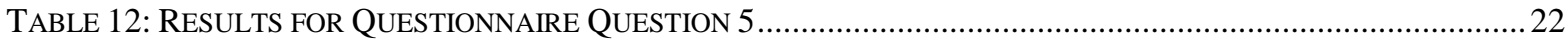

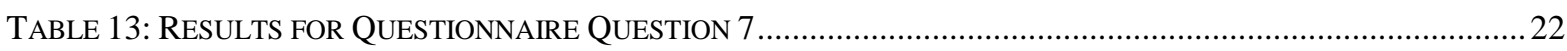




\section{EXECUTIVE SUMMARY}

The use of biometrics to identify individuals has become an important component of efforts to ensure U.S. national security, and has also grown rapidly. Biometrics are, for example, an integral part of the United States Visitor and Immigrant Status Indicator Technology (USVISIT) program. Utilization of biometrics in such systems was mostly a response to urgent security needs, and applications of such technologies were initially limited to state-ofindustry biometric collection devices.

Many risks involved in the operation of these systems have been identified and addressed. Some risks, however, simply cannot be addressed given the current system designs. To address these issues, DHS Science \& Technology (S\&T) commissioned and funded two projects to research technologies that could be used to acquire fingerprints without physical contact. While this novel approach to fingerprint capture solves several challenges posed by the previous generation of scanners, it also creates several new challenges that must be addressed.

The reporting on the two contactless scanners happens in two parts, one part for each scanner. This report is for the second scanner. For the second scanner 60 participants volunteered for the study. Half of the volunteers started with a traditional contact scanner and the other half started with a prototype contactless scanner. They were asked to leave two sets of prints (the four fingers of each hand) three times for each scanner. First with no instructions, secondly after seeing an instructional video, and thirdly with verbal instructions. The participants then followed the same procedure with the second scanner. The order of scanners were counterbalanced across participants.

The results of this study indicate that while contactless fingerprinting technology is viable the prototypes consistently functioned as intended when used correctly - it presents a number of usability challenges. 


\section{INTRODUCTION}

The United States Department of Homeland Security (DHS) is committed to using cuttingedge technologies and scientific talent in its mission to make America safer. The DHS Science and Technology Directorate (S\&T) is tasked with researching and organizing the scientific, engineering, and technological resources of the U.S. and leveraging these resources into technological tools to help protect the homeland. The Homeland Security Advanced Research Projects Agency (HSARPA) Biometrics Detector Program supports this effort.

The use of biometrics to identify individuals has become an important component of efforts to ensure U.S. national security, and has also grown rapidly. Biometrics are, for example, an integral part of the United States Visitor and Immigrant Status Indicator Technology (USVISIT) program. Utilization of biometrics in such systems was mostly a response to urgent security needs, and applications of such technologies were initially limited to state-ofindustry biometric collection devices. These devices included primarily optical and capacitive discharge capture equipment for contact-based electronic fingerprint collection, conducted in a manner similar to ink-based fingerprinting.

Many risks involved in the operation of these systems have been identified and addressed. Some risks, however, simply cannot be addressed given the current system designs. One such risk is the transmission of pathogens by the contact surface of the state-of-industry scanners. Another risk factor identified since wide deployment of biometric systems was the impact of the new biometric sample collection pathways on the existing daily operations: some new collection tasks added anywhere from 15 seconds to over a minute to the normal operations processes, depending on the number of samples being collected. In many cases, the additional time required for biometric collection significantly slowed the throughput of the overall process such collection was intended to support.

To address these issues, DHS S\&T commissioned and funded two projects to research technologies that could be used to acquire fingerprints without physical contact. These technologies utilize structured light illumination and optical spectrum and focus diversity. The goal of such a system is to develop the design basis for a whole new generation of biometric capture devices that can rapidly capture high-resolution images of all 10 fingerprints, without physical contact with the biometric sensor. While this novel approach to fingerprint capture solves several challenges posed by the previous generation of scanners, it also creates several new challenges that must be addressed.

Some of these challenges are related to human factors that affect biometric systems performance. It is necessary to closely examine these human factors issues by studying the usability of new contactless fingerprinting devices in terms of ergonomics and 
anthropometrics, affordance, accessibility, and user satisfaction of non-traditional form factors. Input on human factors and anthropometrics will result in a more robust operational system that increases user performance (timing and quality) and encourages user acceptance. This report describes the usability tests performed on a contactless prototype by the National Institute of Standards and Technology (NIST) biometrics usability team and the NIST Image Group.

According to ISO 9241-11, usability is "the extent to which a product can be used by specified users to achieve specified goals with effectiveness, efficiency and satisfaction in a specified context of use" [5].

- Efficiency is a measure of the resources expended in relation to the accuracy and completeness with which users achieve goals. Efficiency is related to productivity and is generally measured as task time.

- Effectiveness is a measure of the accuracy and completeness with which users achieve specified goals. Common metrics include completion rate and number of errors.

- User Satisfaction is the degree to which the product meets the users' expectations - a subjective response in terms of ease of use, satisfaction, and usefulness.

This study measured a prototype contactless scanner on each of the three dimensions of usability.

\section{METHOD}

\subsection{PARTICIPANTS}

Sixty NIST employees volunteered to participate in this study. There were more men (39) than women (21) in this group, but the ages of the participants were fairly uniformly distributed, as seen in Fig. 1.

Most of the participants were right-handed. Eighty seven percent were right-handed and 13\% were left-handed or ambidextrous. These distributions are very representative of the general population, $87 \%$ of which is right-handed [1]. 


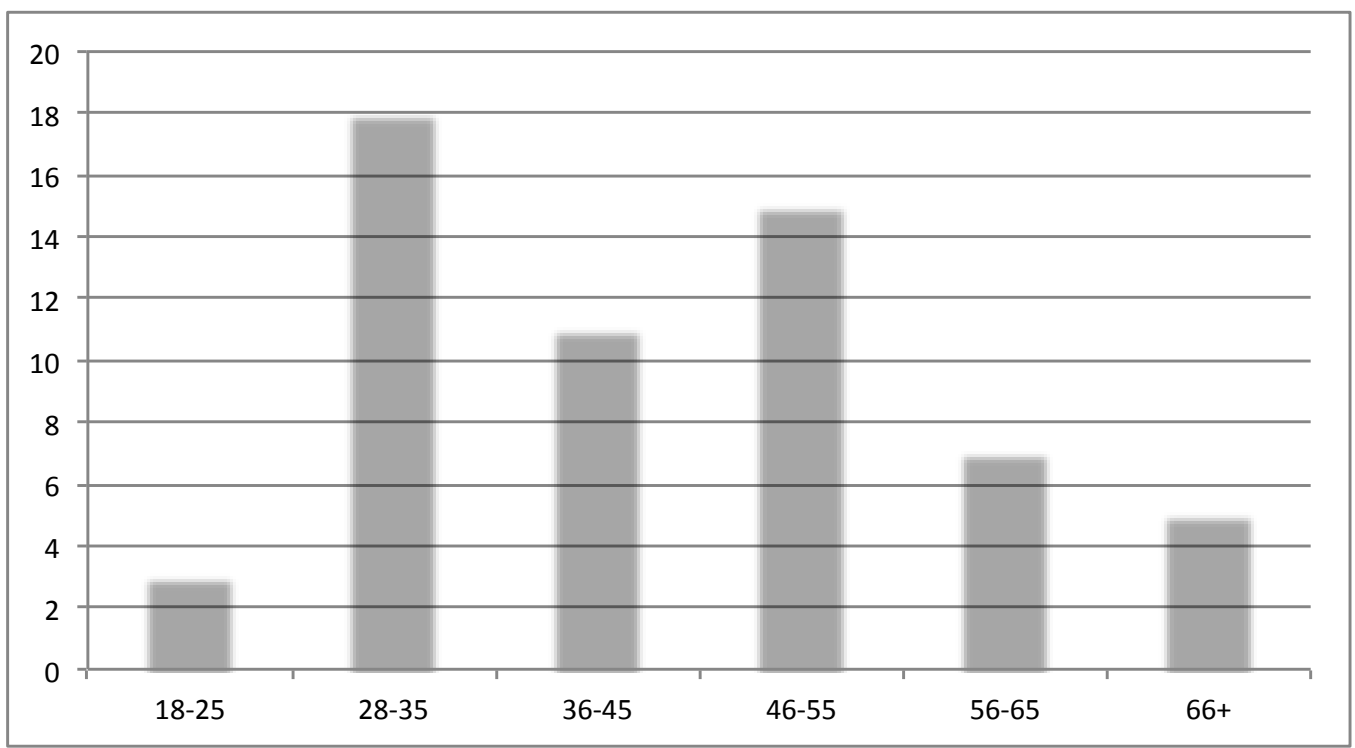

Figure 1: Age Range of Contactless Scanner Participants

\subsection{MATERIALS}

The materials for the test consisted of:

- A contactless fingerprint scanner ${ }^{1}$

- A traditional contact scanner that was positioned on a platform at 20 degrees

- Adjustable tables that allowed for accurate positioning of scanner height

- Floor mats with silhouettes of yellow feet to indicate where participants should stand

- Video instructions

- Custom software

\subsubsection{Contactless Scanner}

The contactless fingerprint scanner ("finger-on-the-fly", FOTF) used, measured $46 \mathrm{~cm}$ (18.11 in) x $35 \mathrm{~cm}(13.78 \mathrm{in}) \times 17 \mathrm{~cm}$ (6.69 in) and had a $5 \mathrm{~cm}$ (1.97 in) x $9.5 \mathrm{~cm}$ (3.74 in)

\footnotetext{
${ }^{1}$ Specific hardware and software products identified in this report were used in order to perform the evaluations described. In no case does such identification imply recommendation or endorsement by the National Institute of Standards and Technology, nor does it imply that the products and equipment identified are necessarily the best available for the purpose.
} 
opening to pass a hand through, between a flange at the top of the device and a shelf beneath. On the shelf was a glass panel that illuminated when ready to collect prints (see Figure 2). A PC monitor attached to the scanner displayed directions and feedback to the participants.

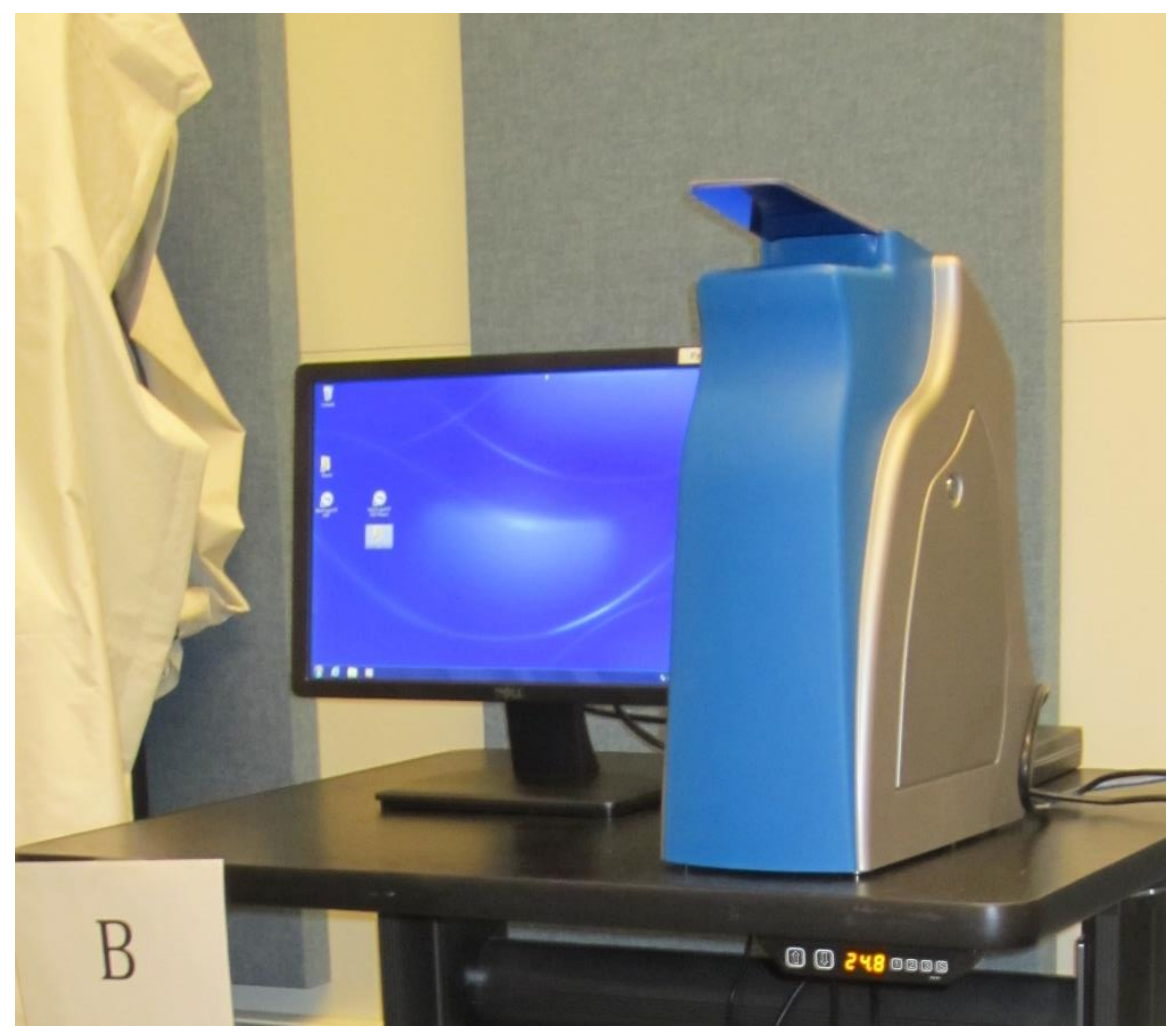

Figure 2: Contactless Fingerprint Scanner

On top of the flange that the participants were to pass their hands under was attached a directional label with a picture and text showing how to use the device (see Figure 3 ). Participants were to pass their hands, one at a time, between the flange and the glass panel. The system would indicate success or failure on the PC monitor. 


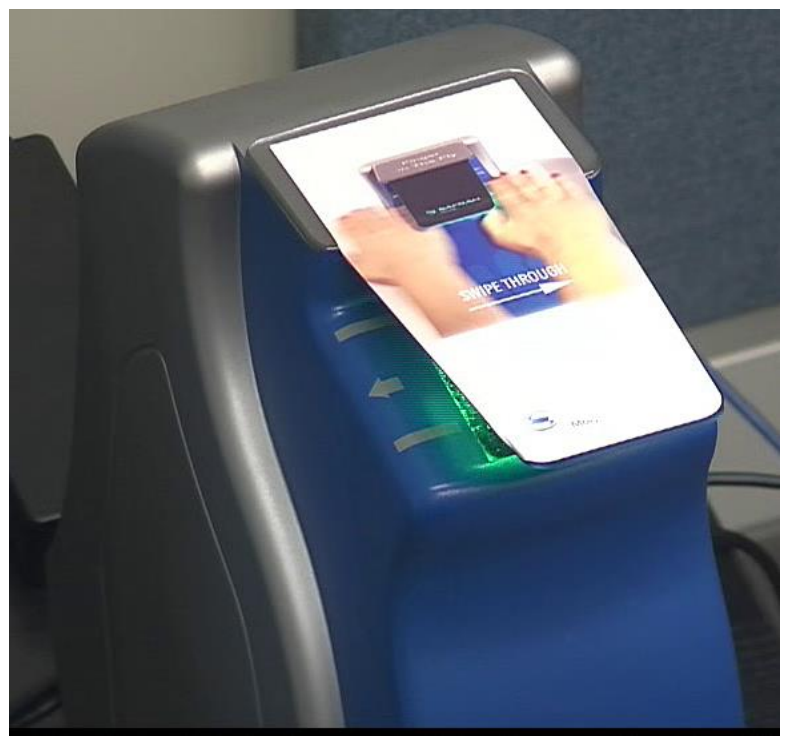

Figure 3: Directional Label on Contactless Scanner

\subsubsection{Contact Scanner}

The traditional contact fingerprint scanner used in this study - shown in Figure 4 - measured approximately $15.2 \mathrm{~cm}(6.0 \mathrm{in}) \times 15.2 \mathrm{~cm}$ (6.0 in) x $15.2 \mathrm{~cm}(6.0 \mathrm{in})$. On top of the scanner was a glass platen: this was the contact surface upon which participants placed their fingers for fingerprint capture. Above the platen was a line of four Light Emitting Diodes (LEDs) at the top edge of the scanner platen surface, each capable of emitting a red or a green light. On each side of the platen were two indicators (four in total) corresponding to a Right Slap, Right Thumb (on the right side), Left Slap, and Left Thumb (on the left side). These indicators would light up to indicate which fingers or thumb the participant was supposed to present. The scanner also emitted audible tones - beeps - whenever it successfully captured a print image. Note that what appears to be a horizontal line or delineation between the left and right icons is inside the scanner and not anything present at the surface of the platen.

A custom capture application was used to control the fingerprint scanner and collect the digital images of participants' fingerprints. The LED lights, slap icons, and audible tones guided participants through the capture process.

When the scanner was ready, all of the LED lights above the platen were red and one of the four indicators on the sides of the platen was illuminated. The sequence in which the indicators were illuminated corresponded to the slap sequence: right four-finger slap and then left four-finger slap. 
LEDs glowed red at the beginning of the process and when the participant's finger position was incorrect; they glowed green when finger positioning was correct

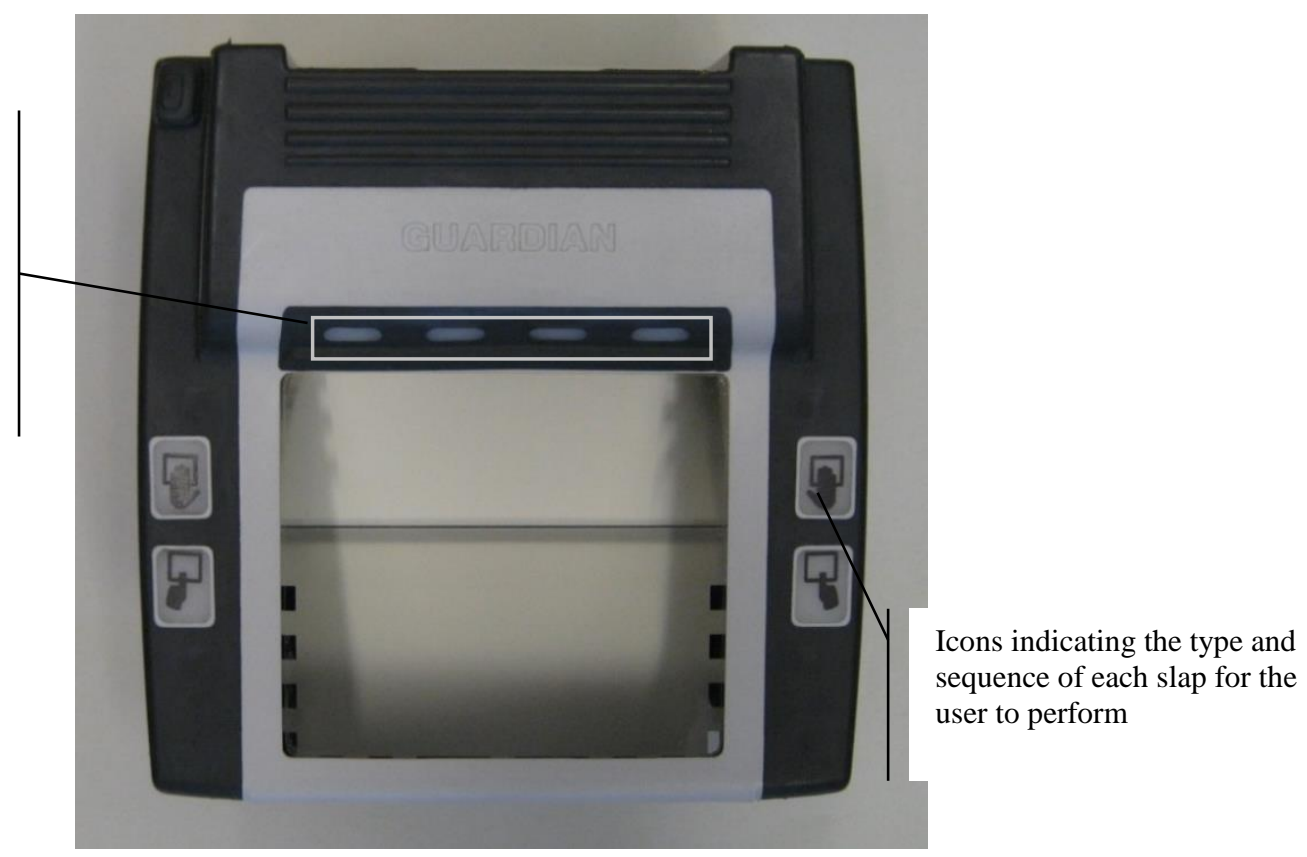

Figure 4: Traditional fingerprint scanner with indicators

Once a participant placed fingers on the platen, the four LED lights indicated whether or not the scanner was able to read the user's fingerprints for the particular slap being requested. When the user performed a right four-finger slap, the corresponding LED lights, e.g., the one on the far left for the index finger, the one on the far right for the little finger, turned from red to green if the scanner was able to read the appropriate fingerprints. Once all four lights turned green - indicating that all the fingers were readable - the scanner software checked the quality of the fingerprint image it was attempting to capture. After a few seconds, if the quality was acceptable, the scanner captured the image and beeped, indicating that it successfully captured an image. If the quality was insufficient, the scanner would stay on the same slap, until a successful capture and then moved to the next slap.

The traditional scanner was placed on an adjustable table and angled at 20 degrees, resulting in a platen surface height of $91.44 \mathrm{~cm}$ (36 in), the recommended platen height (see Figure 5) [3].

\subsubsection{Instructional Materials}

Each of the scanners had an associated instructional video. The videos for the traditional contact scanner were developed by the researchers and depicted (with captioning) what hand to start with, the correct positioning of the fingers, and how to tell when the print had been 
accepted. The instructions were repeated for the other hand. The video for the contactless scanner was provided by the manufacturer. It depicted the starting hand and how to swipe across the glass surface.

\subsection{PROCEDURE}

Before the test began each participant completed the demographic questionnaire shown in Appendix A. Once a participant filled out the demographic questionnaire, he or she was instructed to present his/her fingerprints three times to the traditional scanner and to the contactless scanner, each time under different conditions.

The fingerprint device the participants started with (contact or contactless) was counterbalanced across participants to control for learning effects - i.e., the first participant in the contactless scanner group would use the traditional scanner first and the contactless scanner second for each task, while the second participant would use the scanners in the opposite order, and so on. Once a participant completed the fingerprinting tasks on the first device under the first two conditions they were asked to complete the tasks on the second device under the same two conditions.

All scanners were cleaned in between participants, when needed, to remove any visible fingerprints.

For the first fingerprinting task (Task 1), participants were given no instructions whatsoever about how to use the scanners. Researchers verbally instructed participants to step up on to the mat (shown in Figure 5) "when you believe the device is ready to collect prints" and to step off "when you think the device has collected both sets of prints." The instructional label on contactless scanner was covered so that the participants would have no instructions.

For the second task (Task 2), participants watched one of the instructional videos demonstrating the correct collection procedure for the scanner they were about to use. The instructional label on the contactless scanner was also uncovered. After viewing the video the participants were asked to complete the collection again, following the same verbal instructions used in Task 1.

After each participant completed both Task 1 and Task 2 on both scanners, he or she filled out the post-task questionnaire shown in Appendix B. Afterwards the participants were instructed to leave one more set of prints with each device they had used before (Task 3 ), this time with detailed verbal instructions on how to present prints for the device. These instructions were given before and, if needed, during the attempt 


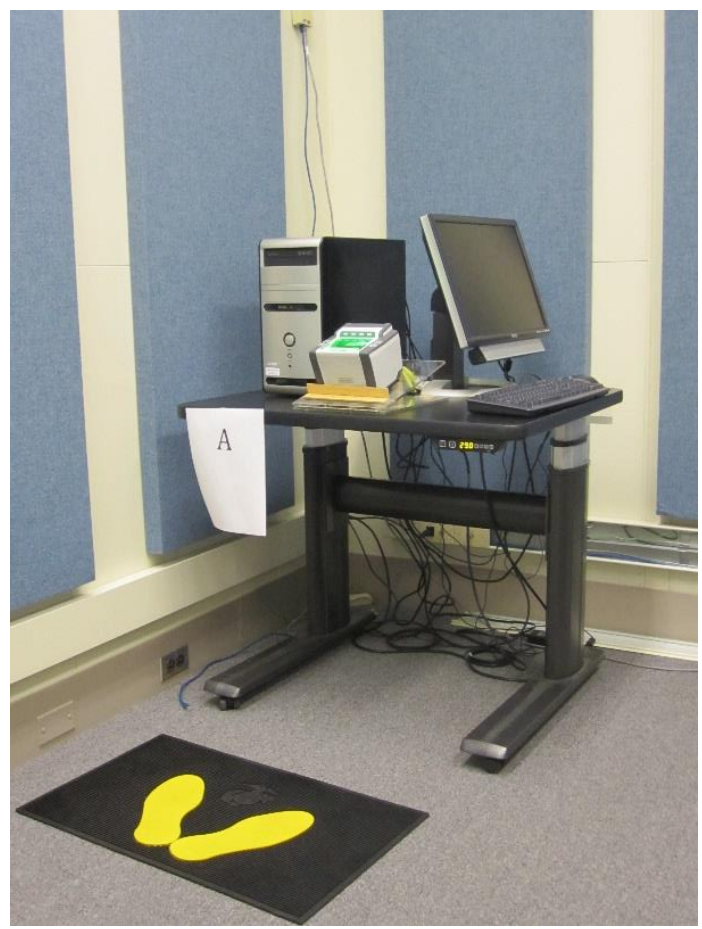

Figure 5: Traditional Fingerprint Scanner Setup Showing Mat and Adjustable Table

\section{RESULTS}

This section describes the results of the usability tests performed on the contactless scanner, with comparisons to the results of tests on the traditional contact scanner used by all participants.

\subsection{USABILITY METRICS}

As described in the introduction to this document, this study was designed to test a contactless fingerprint scanner prototype and measure its usability in terms of efficiency, effectiveness, and user satisfaction. These three dimensions of usability have specific definitions in the context of this study, described in the subsections below.

\subsubsection{Efficiency}

The efficiency of each device was measured in terms of how long it took for participants to complete fingerprinting tasks. Figure 6: Testing Timeline details the testing timeline and sequence of activities for each task. 


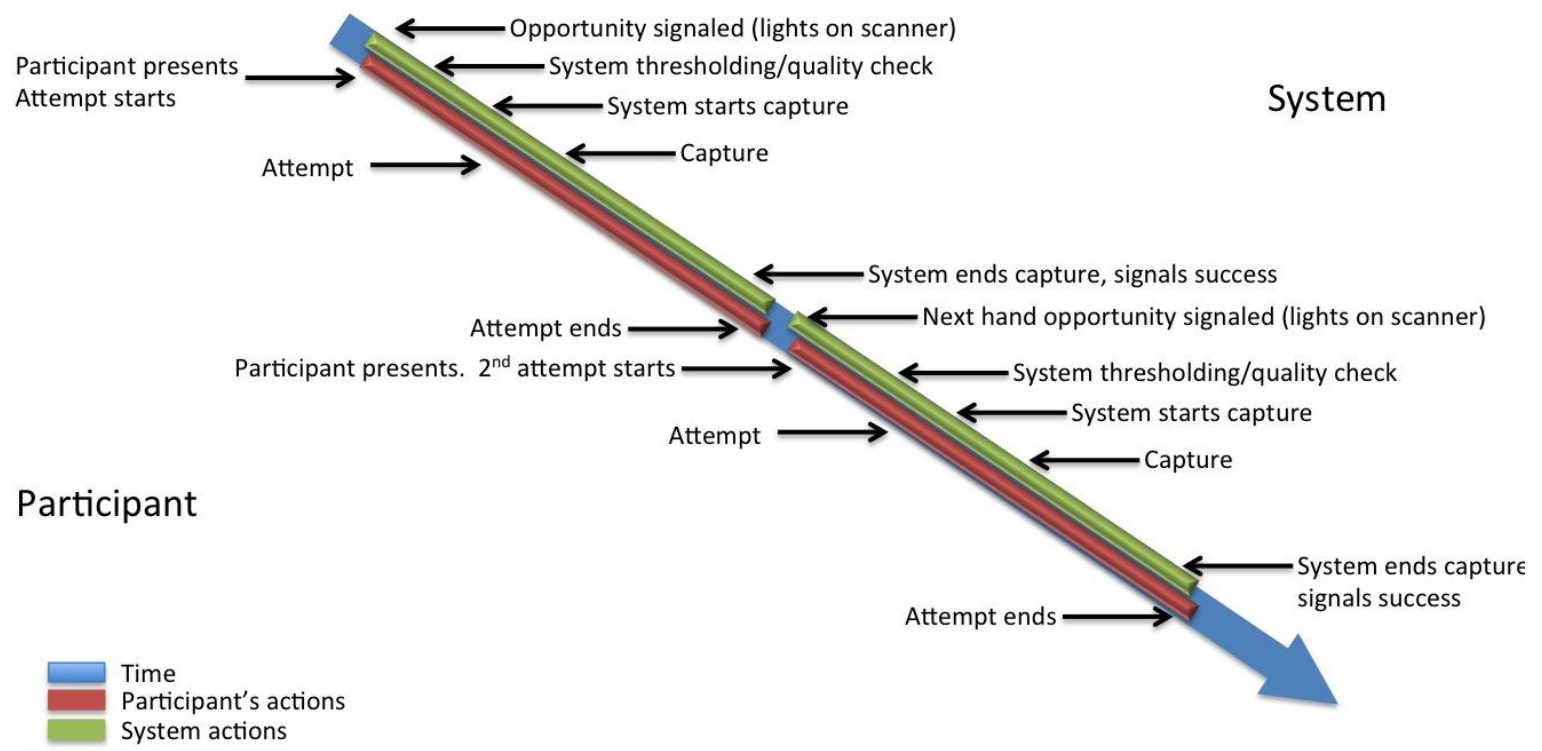

Figure 6: Testing Timeline

\subsubsection{Effectiveness}

The effectiveness of each fingerprinting device was measured in two ways: by task success and by the quality of captured fingerprints.

For purposes of this study, a successfully completed task was one in which the participant presented his or her fingers in such a way that the device was able to capture fingerprint images.

\subsubsection{Print Quality}

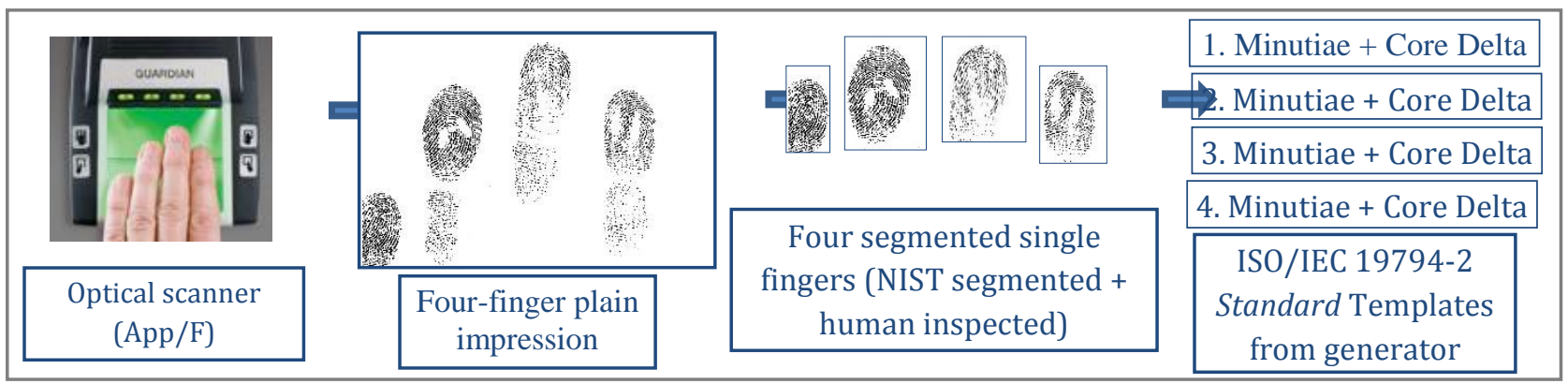

Figure 7 - Data outputs and processing from contact optical scanner 


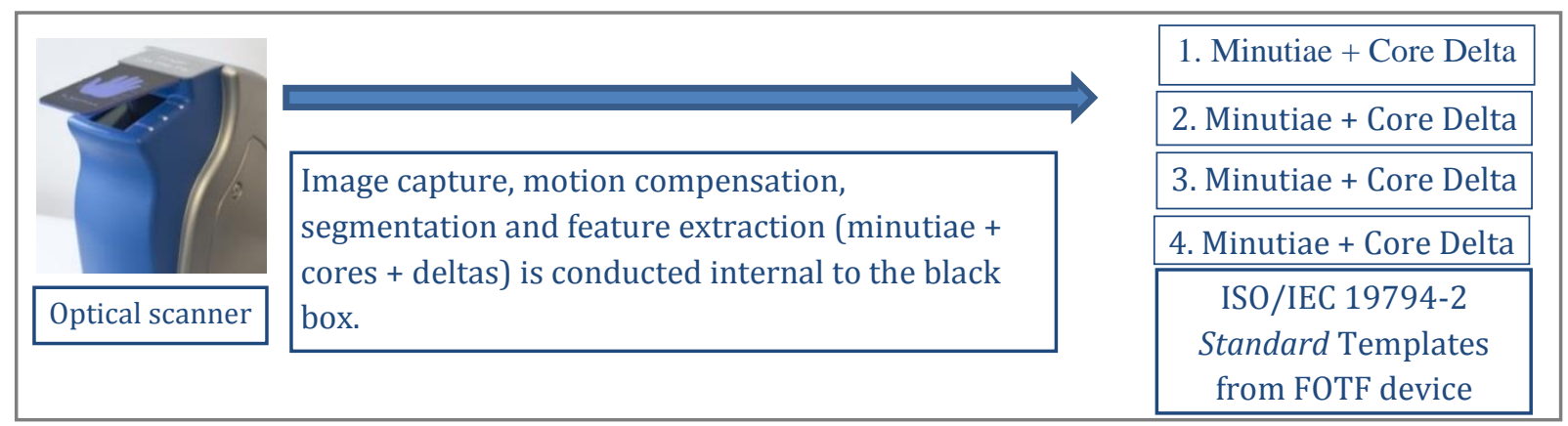

Figure 8 - Data outputs from contactless scanner as configured for NIST trial

\subsubsection{Output data}

As depicted in Figure 8, The contactless Finger-on-the-Fly (FOTF) device was configured to produce templates; it did not emit fingerprint images. The device has the capability to produce images - they are displayed in the client PC application - but the contactless scanner vendor, in discussion with NIST before the test, declined to allow production of images. NIST agreed to proceed. The motivations of NIST and the vendor are discussed as follows:

- The vendor has built a device whose primary intended application is in a rapid physical access control system. As such there would be no requirement to produce or send images anywhere - authentication would be executed internally using fully proprietary fingerprint templates i.e., interoperability is not required.

- On behalf of the US Government, NIST expressed an operational need for interoperability in certain candidate applications. In order to assess interoperability, the vendor agreed to produce standard fingerprint templates. This satisfied the following objectives:

1. To allow measurement of recognition accuracy via comparison of templates output by the FOTF device;

2. To support measurement of interoperability i.e. the accuracy achieved when FOTF templates are compared with templates derived from conventional plainimpression images, in this case a contact optical sensor. That contact sensor has been certified by the FBI under its Appendix F optical imaging specification.

3. To preclude detailed examination of the images, and comparison of those images with plain-impression images using, for example, third party recognition algorithms. The motivation of the vendor here may have been to avoid leakage of intellectual property associated with the imaging mechanism. 
The templates are conformant instances of ISO/IEC 19794-2:2005. This standard has been used in credentialing activities across the world and is being used in India for authentication of UID enrollees. This standard is essentially equivalent to the INCITS 378 standards (2004, and 2009), although different binary header formats mean data would have to be transcoded. The INCITS 378:2004 standard is used for authentication of US Government PIV cardholders.

The templates include minutia points. They also include core and delta information which is encoded in standardized form in the extended data block of ISO/IEC 19794-2:2005. The templates do not include any proprietary (non-standard) data.

\subsubsection{Collected data}

Table 1 - Counts of subjects and presentations used in the analysis

\begin{tabular}{|c|c|c|c|c|}
\hline$\#$ & Quantity & Search & Reference & Total \\
\hline 1. & Number of persons & 60 & 60 & 60 \\
\hline 2. & $\begin{array}{l}\text { Number of persons using the contact } \\
\text { scanner }\end{array}$ & 54 & 60 & 60 \\
\hline & $\begin{array}{l}\text { Number of persons using the contactless } \\
\text { scanner }\end{array}$ & 60 & 60 & 60 \\
\hline 3. & $\begin{array}{l}\text { Number of the contactless scanner } \\
\text { capture events }\end{array}$ & 294 & 120 & 414 \\
\hline 4. & $\begin{array}{l}\text { Number of the contact scanner capture } \\
\text { events }\end{array}$ & 188 & 118 (two single handed) & 406 \\
\hline
\end{tabular}

\subsubsection{Number of fingers}

The device produces between zero and four templates, one from each captured finger. Ordinarily the device will produce four templates; it produces fewer when the human presentation is outside of a capture envelope defined by the physical location of the fingertips and the speed of presentation - sufficient misplacement, or too-fast a hand motion, will inevitably cause failure. The occurrence of such events appears in Table 2. 
Table 2 - Counts of $\mathbf{N}$-finger captures using the contactless scanner

\begin{tabular}{|l|l|l|l|l|l|}
\hline \# & Quantity & $\mathrm{N}=4$ & $\mathrm{~N}=3$ & $\mathrm{~N}=2$ & $\mathrm{~N}=1$ \\
\hline 1 & $\begin{array}{l}\text { Fraction of all captures that } \\
\text { yielded N fingers }\end{array}$ & 0.92 & 0.03 & 0.02 & 0.03 \\
\hline 3 & $\begin{array}{l}\text { Fraction of all reference } \\
\text { captures (i.e. last use) that } \\
\text { yielded N fingers }\end{array}$ & 0.99 & 0.01 & - & - \\
\hline 3 & $\begin{array}{l}\text { Number of people (total 60) } \\
\text { who on at least one occasion } \\
\text { made a presentation that } \\
\text { resulted in N fingers being } \\
\text { captured }\end{array}$ & 60 & 11 & 5 & 4 \\
\hline 4 & $\begin{array}{l}\text { Number of people (total 60) } \\
\text { who in their reference } \\
\text { presentation (i.e. last use) } \\
\text { made a presentation that } \\
\text { resulted in N fingers being } \\
\text { captured }\end{array}$ & 60 & 1 & - & - \\
\hline
\end{tabular}

In all cases the contact scanner captured all four fingers.

To summarize the contactless scanner performance, the following statements hold.

- All persons were able to execute a complete four-finger presentation to FOTF.

- Complete four-finger presentations were more common after the initial usage period.

\subsubsection{Speed of capture}

Table 3 - Durations of N-finger captures using the contactless scanner

\begin{tabular}{|c|c|c|c|c|c|}
\hline \# & Quantity & $\mathrm{N}=4$ & $\mathrm{~N}=3$ & $\mathrm{~N}=2$ & $\mathrm{~N}=1$ \\
\hline 1.1.1.1.1. & $\begin{array}{l}\text { Median duration of captures which yielded only } \mathrm{N} \\
\text { fingers (seconds) }\end{array}$ & 1.4 & 1.9 & 1.9 & 0.4 \\
\hline
\end{tabular}




\begin{tabular}{|l|l|l|l|l|}
\hline $\begin{array}{l}\text { 1.1.1.1.2. } \\
\text { Median duration of reference presentation } \\
\text { captures }\end{array}$ & 1.2 & 5.5 & - \\
\hline
\end{tabular}

The contactless device produces a timing log from each capture. With millisecond resolution, the sensor records the "Finger detected" time and the "Image Acquired" time. The time elapsed between these events is the core functional time of the device. Our best estimate of this duration is $(1.2 \pm 0.2)$ seconds for reference enrollments. It is highlighted, in Table 3. This is the median over 120 captures of left and right hands of 60 persons executing their "reference" enrolment. We consider this duration to be an approximation of that which would apply for habituated individuals i.e. those with prior experience and instruction in using the sensor. In this case this experience amounted to just several uses of the device over several minutes. The median duration of those early-attempt captures was $(1.6 \pm 0.3)$ seconds, with several presentations being made slowly and tentatively.

This duration is the technical component of the entire human-sensor transaction. In an operational setting, an end-to-end transaction time will include this duration and additional human presentational times (approach to the sensor, raising and moving the hand), and any additional system times (for network transmission, door activation etc.). The device can operate in standalone mode, but will often be integrated into a physical access control system or an identity management system. This may extend to remote matching of the captured fingerprint data. Processing times of those components may be additional to the core device times listed here.

\subsubsection{Accuracy and interoperability}

Recognition accuracy is assessed using fingerprint samples collected from the 60 person volunteer crew. This was done offline, in a separate phase after completion of the data collection. Since a primary use of the contactless scanner is for access control, accuracy is stated in terms of one-to-one verification performance.

- Samples: The two recognition error rates, false non-match rate (FNMR) and false match rate (FMR), are computed by comparing samples. A sample in this test is usually a set of four standard fingerprint templates corresponding to the minutiae, core and delta information extracted from each of four fingers. Occasionally fewer than four fingers were imaged, as discussed previously. FMR is estimated by executing "impostor" comparisons of any two samples from different people. This involves comparing left and right hands with all other left and right hands. FNMR is estimated by executing "genuine" comparisons of samples from the same hand of the same person. In the results that follow, the verification samples are the samples 
collected during the initial encounters with the device, and the reference samples are the last ones collected, one from each hand.

- Algorithm: Comparisons are conducted using the Morpho verification software submitted to NIST for this test. This software accepts two samples and produces a single score. Algorithms from other providers could be applied to the samples because the data is in standardized template form (ISO/IEC 19794-2:2005).

- Scores: In all cases comparison of sample A with sample B produces a single matcher score. Internally, the algorithm compares $\mathrm{N}=1 \ldots 4$ fingerprint templates that constitute sample A with the $M=1 \ldots 4$ fingerprint templates that constitute sample $\mathrm{B}$, and then fuses the result. While the fusion method is proprietary our trials indicate that software implements a maximum of the NM scores. High scores are more indicative that the two samples come from the same hand.

- Single finger comparison: This study did not execute single-finger matching because the FOTF device natively collects and operates on four-finger data.

- Threshold: We fix a threshold value so that we can state FNMR. Any moderately high threshold could be used. We chose the value of 2891 which gives FMR $=0.001$ over the nearly 500000 impostor comparisons. The use of samples from only 120 hands means that this FMR value would vary substantially with larger populations.

The accuracy results are summarized in Table 4. The Table shows false non-match rates obtained by comparing all "verification samples" with "reference samples" as described above.

\section{Table 4 - Cross sensor false non-match rates}

$\operatorname{FNMR}(T)$ at $\mathrm{T}=2891$.

Verification sensor

contact plain optical contactless

Reference

(enrolment) sensor contact plain optical

contactless
0.02

0.00
0.03

0.06

The notable observation here is that the cross-device error rate can be zero. This indicates that samples from the plain-impression scanner can be verified against contactless enrollments at this threshold. Unfortunately the symmetric entry indicates that some 
contactless samples do not verify against the optical enrolment; this arises because the early presentations were made without any subject instruction.

The higher contactless - contactless error rate, 0.06 , is unexpected since a device can interoperate with itself, by definition. The reason for the higher value is probably the presence of samples from non-conformant (too slow, too fast) presentations. Also, there is considerable uncertaint ${ }^{2}$ associated with the numbers in this table due to small population size $^{2}$. Moreover, the errors that do occur are almost certainly not due to innate defectiveness of fingers, nor permanent inability of the device. This is evident from Table $\mathbf{5}$ which shows every subject can authenticate using any pair of sensors, although several attempts may have been necessary.

\section{Table 5 - Number of persons unable to authenticate over repeated attempts}

\begin{tabular}{|l|l|l|l|}
\hline & & \multicolumn{2}{l|}{ Verification sensor } \\
& & contact plain optical & contactless \\
\hline $\begin{array}{l}\text { Reference } \\
\text { (enrolment) sensor }\end{array}$ & contact plain optical & 0 & 0 \\
\cline { 2 - 4 } & contactless & 0 & 0 \\
\hline
\end{tabular}

\subsubsection{Conclusions and Recommendations}

The contactless device is an advanced optical design capable of collecting biometric data that can be authenticated. As with most biometric sensors, if the subject makes a presentation that does not conform to that intended by the system designers, a poor sample can result and recognition failure can ensue.

Based on template comparison of a small number of examples, the device appears to have some level of interoperability with the traditional optical scanners used to capture plain-impressions. More analysis is required to further assess its interoperability with legacy devices.

2 The uncertainty is determined by the number of individuals (60), the number of samples $(\approx 400)$, and the number of trials $(\approx 500,000)$. The trials are not independent so the uncertainty is largely dependent on the number of individuals. Given this population size, the measured error rates have uncertainty of nearly $50 \%$ (via the "Rule-of-30", 95\% confidence). 
Moreover, it seems appropriate to further test the device in order to better characterize performance indicators such as recognition error rates, failure-to-capture / re-attempt rates, and overall system human transactional throughput. Such trials might recruit and prepare populations who are representative of users at two stages:

1. The population who would encounter the device just after it was first deployed, and

2. The population who had significant prior use, or exposure to the device, either by being specifically instructed, or by watching other people using the device

Such a test would allow computation of error rates that at the beginning and end of an interval that brackets the initial introduction of a device (into airports, say), and the end-state where the device has been used over the long term. The test should include larger population sizes. This is necessary to get a higher fidelity estimate of error rates, and, particularly, re-try rates - the number of attempts needed to authenticate. The test should also collect plainimpression samples from Appendix F certified optical scanners.

\subsubsection{User Satisfaction}

In this study, user satisfaction was measured by whether, and to what extent, participants found a given device intuitive, easy to use, fast, and good at providing feedback. User satisfaction data was collected through the use of the post-task questionnaire in Appendix B.

There were five questions to which participants responded with a binary (e.g., yes/no) answer and comments, as well as two questions to which they responded with comments only.

Participants provided two sets of responses per question, one set for the contact scanner and one set for whichever contactless scanner they used.

\subsection{CONTACTLESS SCANNER}

The organization of this section is similar to that of the previous section detailing the results of the tests on the contactless scanner.

\subsubsection{Efficiency}

The average amount of time it took for participants to complete fingerprinting tasks with the contactless scanner and the contact scanner are shown in Table 6 below. 


\subsection{CONTACTLESS SCANNER}

The organization of this section is similar to that of the previous section detailing the results of the tests on the contactless scanner .

\subsubsection{Efficiency}

The average amount of time it took for participants to complete fingerprinting tasks with the contactless scanner and the contact scanner are shown in Table 6 below.

Table 6: Average Task Times in Seconds - Contactless Scanner

\section{Time to Complete Task (in seconds)}

\begin{tabular}{l|c|c|c} 
& $\begin{array}{c}\text { Task 1 (no } \\
\text { instructions) }\end{array}$ & Task 2 (video) & $\begin{array}{c}\text { Task 3 (verbal } \\
\text { instructions) }\end{array}$ \\
\hline Contact Scanner & 26.78 & 17.42 & 18.84 \\
\hline Contactless Scanner & 48.49 & 16.88 & 13.40
\end{tabular}

As Table 6 shows, it took longer, on average, for participants to complete Task 1 on the contactless scanner than on the contact scanner. However, the participants took less time to complete Tasks 2 and 3 - for which they had video and verbal instructions, respectively - on the contactless scanner than they did on the contact scanner.

\subsubsection{Effectiveness}

Table 7 shows the number of participants who had their prints collected by each device in Task 1, Task 2, and Task 3. Participants were more successful with the contact scanner than with the contactless scanner for Task 1. In Tasks 2 and 3, participants had roughly the same success rate with both scanners. 
Table 7: Successful Print Collection per Task - Contactless Scanner

\# of participants (out of 58) who successfully completed the fingerprinting task

\begin{tabular}{l|c|c|c} 
& Task 1 (no instructions) & Task 2 (video) & $\begin{array}{c}\text { Task 3 (verbal } \\
\text { instructions) }\end{array}$ \\
\hline Contact Scanner & 44 & 53 & 58 \\
\hline Contactless Scanner & 11 & 52 & 58
\end{tabular}

\subsubsection{User Satisfaction}

In general, an equal number of participants found the contactless scanner as easy to use as the traditional contact scanner. Participants were roughly equally split in terms of which scanner they preferred, in spite of the fact that more participants said that it was easier on the contact scanner to decipher the lights, determine which hand was expected, tell when the device was ready to accept prints, and to tell when prints were accepted.

\section{Which of the scanners did you find easier to use? Why?}

Twenty-six participants said they found the contact scanner easiest to use, while 30 said they found the contactless scanner easier to use. Two participants said they found neither scanner easier to use than the other.

Table 8: Results for Questionnaire Question 1

\begin{tabular}{c|c|c} 
Contact Scanner & Contactless Scanner & Neither \\
\hline 26 & 30 & 2
\end{tabular}

Participant comments included:

- Contactless scanner was significantly easier to use once I had seen the video

- It made sense to lay your fingers on something (for contact)

- Contact scanner is easier to use with no instructions, contactless scanner is easier to use with instructions

Did the scanner lights mean anything to you? 
Fifty-five of the participants said that the lights on the contact scanner had some meaning (1 after seeing the instructional video): 3 said that the lights had no meaning for them. For the contactless scanner, 30 participants said they believed the lights on the device indicated something - 1 provided an incorrect interpretation of what the lights meant - and 25 participants said the lights had no meaning for them. Three participants indicated that they had not noticed the lights on the contactless scanner at all.

Table 9: Results for Questionnaire Question 2

\begin{tabular}{r|c|c|c|c|c} 
& Yes & No & $\begin{array}{c}\text { Yes After } \\
\text { Video }\end{array}$ & Wrong* & $\begin{array}{c}\text { Didn't Notice } \\
\text { Lights }\end{array}$ \\
\hline Contact Scanner & 54 & 3 & 1 & 0 & 0 \\
\hline $\begin{array}{r}\text { Contactless } \\
\text { Scanner }\end{array}$ & 29 & 25 & 0 & 1 & 3
\end{tabular}

\begin{abstract}
* This means that the participants provided an incorrect interpretation of what the lights on the scanner indicated.
\end{abstract}

Participant comments included:

- Maybe there should be some indicator light to say "ready to go" (for contactless)

- That's where I should press my hand down. Which was wrong (for contactless)

- $\quad$ Lights green $=$ go (for both scanners)

\title{
Could you tell what hand the scanner was expecting?
}

For the contact scanner, the majority of participants (47) said that they could tell which hand the device was expecting them to present ( 1 after seeing the video). 9 participants said that they were unable to tell which hand the contact scanner was expecting. One participant said that he/she was "somewhat" able to tell which hand the device expected, and one other participant said that it didn't matter which hand was presented to the scanner first.

Only 11 participants said that they could tell which hand the contactless scanner was expecting: 43 said they could not tell at all. Three participants said they were "somewhat" 
able to tell. Again, one participant said it didn't matter which hand was presented to the scanner first.

Table 10: Results for Questionnaire Question 3

\begin{tabular}{l|c|c|c|c|c} 
& Yes & No & $\begin{array}{c}\text { Yes After } \\
\text { Video }\end{array}$ & Somewhat & $\begin{array}{c}\text { Didn't } \\
\text { Matter }\end{array}$ \\
\hline Contact Scanner & 46 & 9 & 1 & 1 & 1 \\
\hline Contactless Scanner & 11 & 43 & 0 & 3 & 1
\end{tabular}

Participant comments included:

- I was not certain but I thought the right hand should be scanned first (for contact)

- I couldn't tell what hand was expected or when it was done gathering data (for contactless)

\section{Could you tell when the scanner was ready to accept a print?}

Forty-nine participants said that they could tell when the contact scanner was ready to accept a print (i.e., for them to present a hand for fingerprinting): 9 participants said that they could not tell. Roughly half of the participants (29) said that they could tell when the contactless scanner was ready to accept a print, and 2 of them were only able to do so after seeing the video. The other half (29) were unable to tell when the contactless scanner was ready to accept prints.

Table 11: Results for Questionnaire Question 4

\begin{tabular}{l|c|c|c} 
& Yes & No & Yes After Video \\
\hline Contact Scanner & 49 & 9 & 0 \\
\hline Contactless Scanner & 27 & 29 & 2
\end{tabular}

Participant comments included:

- I basically guessed it was ready (for contactless)

- It was very small to see (for contact) 


\section{Could you tell whether the print was successfully captured?}

Most (51) participants said that they could tell when the contact scanner successfully captured their fingerprints, although 4 said they were unable to do so until they watched the instructional video. One participant reported being "sort of" able to tell when the scanner actually captured prints, and the rest (6) were unable to tell at all.

For the contactless scanner, 40 participants said that they were able to tell when the scanner had captured their prints ( 2 after seeing the video). As with the contact scanner, one participant reporting being "sort of" able to tell when the prints were captured. The other 17 participants said that they could not tell when the prints had been captured.

Table 12: Results for Questionnaire Question 5

\begin{tabular}{l|c|c|c|c} 
& Yes & No & Yes After Video & Sort Of \\
\hline Contact Scanner & 47 & 6 & 4 & 1 \\
\hline Contactless Scanner & 38 & 17 & 2 & 1
\end{tabular}

Participant comments included:

- Yes but less efficiently than contact scanner

- The "beep" was helpful (for contact scanner)

- Yes, but only if you look at the screen (for contactless scanner)

\section{What scanner did you prefer? Why?}

Participants were roughly evenly split in terms of which scanner they preferred: 28 preferred the contact scanner, 27 preferred the contactless scanner, and 1 preferred neither.

Table 13: Results for Questionnaire Question 7

\begin{tabular}{c|c|c} 
Contact & $\begin{array}{c}\text { Contactless } \\
\text { Scanner }\end{array}$ & Neither \\
\hline 28 & 27 & 1
\end{tabular}

Participant comments included: 
- (contactless) took less time and easily collected my fingerprints

- I'd rather not touch the glass especially if this is a public workstation

- Could use it easily without the video (for contact)

- Better indication the process was completed (for contact)

\subsubsection{Observations}

Many participants - 44 out of 48 - attempted to touch the glass surface on the contactless scanner when they performed their first fingerprinting task without any instructions, rather than swiping their hands over it. ${ }^{3}$ Since 22 of these participants interacted with the contactless scanner first, they could not have been influenced by using the contact scanner.

A significant number of participants also appeared to be confused by the conflicting markings on the contactless scanner. Fifteen of them mentioned that, as shown in Figure 3, the arrow on the instructional label points in the opposite direction from the one printed on the device.

\section{DISCUSSION}

The results of this study indicate that while contactless fingerprinting technology is viable the prototypes consistently functioned as intended when used correctly - it presents a number of usability challenges.

\subsection{MENTAL MODELS OF FINGERPRINTING - TOUCHING THE GLASS}

During Task 1, when they were working without instructions, many participants placed their hands on the glass surface of the contactless scanner. As noted in Sec. 3.3.4, participants very frequently did this regardless of whether or not they used the contact scanner before using a contactless scanner. This indicates that people's mental model (or at least the participants' mental model) of fingerprint collection involves pressing their fingers against a surface. Touching a glass surface seemed to be deeply ingrained into the study participants' idea of

3 Cleaning the glass surface of contactless scanner two proved to be somewhat difficult given the limited space to move and the necessity of using a microfiber lens cloth. 
what fingerprint collection entails - a perception that may be influenced by depictions of fingerprint scanning in popular media and past experiences with electronic fingerprinting.

Fingerprinting Sequence Cues

A usability challenge common to fingerprint scanners of any type - including the contactless scanner prototypes used in this study - is that of informing the user where they are in the fingerprinting process (see Fig. 11 for the fingerprinting task timeline). A fingerprint collection device needs to clearly indicate:

1. readiness to accept prints;

2. which hand is expected (right or left);

3. appropriate hand/finger placement;

4. successful print acquisition; and

5. how to recover from a failed attempt.

The contactless scanner provided cues to the participants almost entirely through the PC monitor (other than the glass panel illuminating green when the device was ready to capture prints). The monitor indicated when the device was ready, how to swipe one's hand through the "active" space where fingerprints could be captured, and whether prints were successfully captured. This dependency on a monitor to display all directions to the user will have to be taken into consideration when deploying this device in an operational setting.

\subsection{HAND INDICATION (RIGHT OR LEFT)}

The contactless scanner indicated what hand was expected by its video. The video showed a right hand swiping across the scanner's glass surface. This video looped showing the right hand swipe until the participant used the scanner. This looping caused some confusion about whether the left hand should be scanned since the video only showed the right hand.

\section{CONCLUSIONS}

The persistent mental model of an electronic fingerprinting process requiring the user to touch a glass surface has two major implications for DHS if the organization plans to continue developing contactless fingerprint technology. First, a concerted effort needs to be made to educate the public in the use of contactless scanners. This means using a number of 
different media - such as video, posters, and Web information - to communicate the same message about how contactless scanners work and how to operate them.

Second, it should be accepted, and planned for, that people will touch any clear, flat surface on a contactless fingerprint scanner and that surface will need to be cleaned. During pilot testing of the scanners prior to the usability study, the research team discovered that it did not take long for the latent prints left on the contactless scanner's glass surface to interfere with the collection of live prints. ${ }^{4}$ This means that in any operational concept of the scanner tested during this study, there will have to be some procedures to clean them.

Educating the public on the proper way to use contactless scanners and keeping the glass surfaces clean enough to capture prints will take time. This time will have to be accounted for when planning throughput numbers for any fingerprinting process involving contactless scanners. It is likely that while people are still growing accustomed to contactless scanners, it will take longer to successfully collect fingerprints, leading to longer lines at entry points.

4 This did not present as much of a problem during actual testing, as all fingerprinting devices were cleaned so as not to leave any prints that might serve as cues of what to do with the scanner. 


\section{REFERENCES}

[1] “Advanced Data From Vital and Health Statistics", US Department of Health and Human Services Centers for Disease Control and Prevention, October 27, 2004, http://www.cdc.gov/nchs/data/ad/ad347.pdf

[2] Tabassi, E., Wilson, C., \& Watson, C. U.S. Department of Commerce, National Institute of Standards and Technology (NIST). (2004). Fingerprint image quality (NIST IR 7151). Retrieved from http://www.nist.gov/manuscript-publicationsearch.cfm?pub_id=905710

[3] Theofanos, M., Orandi, S., Micheals, R., Stanton, B., \& Zhang, N. F. Department of Commerce, National Institute of Standards and Technology (NIST). (2007). Effects of scanner height on fingerprint capture (NIST IR 7382). Retrieved from http://www.nist.gov/manuscript-publication-search.cfm?pub_id=50903

[4] Theofanos, M., Stanton, B., Orandi, S., Micheals, R., \& Zhang, N. F. Department of Commerce, National Institute of Standards and Technology (NIST). (2006).

Usability testing of ten-print fingerprint capture (NIST IR 7403). Retrieved from http://www.nist.gov/manuscript-publication-search.cfm?pub_id=51123

[5] ISO 9241-210:2010 Ergonomics of human-system interaction - Part 210: Humancentred design for interactive systems 


\section{APPENDIX A: DEMOGRAPHICS QUESTIONNAIRE}

National Institute of Standards and Technology (NIST)

Information Technology Laboratory (ITL), Information Access Division (IAD)

Contactless Fingerprint Capture Study Task Evaluation

\section{Demographic Questionnaire}

1. Age:

2. Gender:

Female __ Male

3. Handedness: __ Right handed Left handed Ambidextrous

4. Height: 
APPENDIX B: POST-TASK QUESTIONNAIRE

\author{
National Institute of Standards and Technology (NIST) \\ Information Technology Laboratory (ITL), Information Access Division (IAD) \\ Contactless Fingerprint Capture Study Task Evaluation Post-Task Questionnaire
}

Based on your experience from the tasks with the fingerprint scanners, please answer the following questions.

1. Which of the scanners did you find easier to use? Why?

2. Did the lights on the scanners mean anything to you?

a. Scanner A:

b. Scanner B: 
3. Could you tell what hand the scanner was expecting?

a. Scanner A:

b. Scanner B:

4. Could you tell when the scanner was ready to accept a print?

a. Scanner A:

b. Scanner B:

5. Could you tell whether the print was successfully captured?

a. Scanner A:

b. Scanner B: 
6. What improvements would you make to the scanners? Why?

a. Scanner A:

b. Scanner B:

7. What scanner did you prefer? Why?

\section{Do you have any additional comments? (Do not include any personally identifiable} information in your comments)

NISTIR 8159

Page 30

$10 / 2014$ 